\title{
ARTICLE
}

\section{Test of a Fiber Optic Scintillation Dosimeter with BGO Tip in a ${ }^{60} \mathrm{Co}$ Irradiation Chamber}

\author{
Min Woo SEO, Jae Kyoung KIM, and Jae Woo PARK* \\ Jeju National University, 1 Ara-dong, Jeju-si, Jeju-do 690-756, Korea
}

\begin{abstract}
Following a previous feasibility study, a photon dosimeter have been constructed by attaching a small piece of BGO scintillator to a low attenuation plastic optical fiber for which the light output is measured with a current-type PMT. The dosimeter model is tested in a ${ }^{60} \mathrm{Co}$ irradiation chamber in the dose rate range varying from about 50 to $1200 \mathrm{~Gy} / \mathrm{h}$. The dose rates are measured at a number of predetermined positions in the form of the net PMT current. MCNPX simulations are carried out to calculate the energy being deposited in the scintillator piece at the same positions. The normalized PMT current distribution is compared with that of the deposited energy distribution to evaluate how accurately the dosimeter model can follow the dose rate change. In addition, commercially available alanine pellet dosimeters are used to measure the absorbed dose rates at the same positions. The normalized PMT current distribution is compared with that of the alanine-measured dose rate. A dose conversion factor is obtained at each position by dividing the alanine-measured dose rate by the PMT current and then an average dose conversion factor is calculated from the point values. When the average conversion factor is used, the maximum deviation between the dose rates measured with the dosimeter model and alanine dosimeters, respectively is estimated to be less than $10 \%$. The scintillator piece is irradiated to a dose of $100 \mathrm{kGy}$ with a dose rate of about $4,430 \mathrm{~Gy} / \mathrm{h}$. The reduction of the light output immediately and one day after the irradiation is estimated. The light output decreased to $83 \%$ just after the irradiation and recovered to $95 \%$ one day after the irradiation.
\end{abstract}

KEYWORDS: Fiber optic scintillation dosimeter, BGO scintillator tip, current type PMT, Dose conversion factor

\section{Introduction}

Real-time remotely measuring radiation dosimeters with high spatial resolution are useful to monitoring radiation doses around an irradiator or inside a human phantom for radiation cancer treatment planning. In order to have a higher spatial resolution, a smaller size of the sensor is preferred. Farmer type ionization chambers or MOSFET (Metal Oxide Semiconductor Field Effect Transistor) dosimeters are frequently used for the aforementioned purpose. Farmer type ionization dosimeters including the connecting cables usually have limited spatial resolution. MOSFET dosimeters have excellent spatial resolutions and are convenient for simultaneous measurement at multiple points, but their life times are limited so that their uses are impractical in a high radiation field.

A radiation dosimeter constructed with a small piece of scintillator combined with a light-guiding optical fiber can be a good choice overcoming these limitations all together. A number studies have been conducted on photon dosimeters based on the fiber-optic scintillator concept. Nowotny ${ }^{1)}$ carried out a study on a photon dosimeter constructed with a combination of LiF:W scintillator tip and plastic optical fiber for diagnostic radiology. Lee et $\mathrm{al}^{2{ }^{2}}$ investigated the scintillating optical powers of a radiation sensor fabricated with several kinds of inorganic scintillator tips and a plastic optical fiber with two different lengths. Bedder et al. ${ }^{3)}$ proposed a miniature scintillator-fiberoptic-PMT detector system for the dosimetry of small fields in stereotactic radiosurgery. The detector system consists of a plastic

*Corresponding Author, E-mail:jwpark@jejunu.ac.kr

(C) Atomic Energy Society of Japan scintillator optically attached to a radiation-resistant silica fiber with $400 \mu \mathrm{m}$ core diameter and a head-on-type PMT. In a previous work $^{4}$, we conducted a feasibility study on a fiber-optic photon detector with a BGO scintillator tip and a pulse type PMT, which was based on measuring the pulse counts as a function of radioactivity. While this dosimeter detector produced reasonable data for low activity gamma sources, it gave meaningless results in a high activity radiation field. In order to apply the detector to measuring radiation doses in higher activity photon fields, we have replaced the pulse type PMT with a current type PMT.

In this work, we present the test result of a fiber-optic photon dosimeter composed of a BGO scintillator piece, a light-guiding plastic optical fiber and a current type PMT. The sensor is constructed by attaching a small piece of BGO scintillator, having a volume of $4.9 \times 4.9 \times 10 \mathrm{~mm}^{3}$, to a low attenuation plastic optical fiber of $3 \mathrm{~mm}$ diameter and $10 \mathrm{~m}$ length. We have chosen the BGO scintillator since it is relatively cheap and easily fabricable in any form in the open air. It offers high detection efficiency for gamma-rays due to the large atomic number of bismuth component and the high density, so that the sensor volume can be made smaller.

The dosimeter model is tested in a panoramic ${ }^{60} \mathrm{Co}$ irradiation chamber in the dose rate range varying from about 50 to $1200 \mathrm{~Gy} / \mathrm{h}$ by changing the distance between the dosimeter and the source. MCNPX simulations and alanine pellet dosimeters are used to evaluate the performance of the dosimeter model.

\section{Experiments}

\section{Construction of the model dosimeter}


As shown in Fig. 1, the dosimeter model is composed of scintillator piece, light guiding optical fiber, PMT module, data acquisition unit (DAU) and PC. The scintillator is BGO having a chemical composition of $\mathrm{Bi}_{4} \mathrm{Ge}_{3} \mathrm{O}_{12}$, which has been purchased in cubical form having a volume of $10^{3} \mathrm{~mm}^{3}$ from the Institute for Single Crystals (ISC), Ukraine. Its density is $7.13 \mathrm{~g} / \mathrm{cm}^{3}$ and the refractive index is 2.15 . Its light yield is given as $20 \%$ that of $\mathrm{NaI}(\mathrm{Tl})$ and the wavelength of peak emission is $480 \mathrm{~nm}$. The scintillator piece is fabricated by cutting the cube into four equally sized pieces and then polishing them with a series of polishing media including $0.5 \mu \mathrm{m}$ alumina powder, the final volume being $4.9 \times 4.9 \times 10 \mathrm{~mm}^{3}$. It is wrapped with a Teflon tape to reduce the light escape from the piece. The light guiding optical fiber, which is a product (PGR-FB3000) of Toray Industries, Inc., has $3 \mathrm{~mm}$ diameter and $10 \mathrm{~m}$ length. The core of the fiber is made of PMMA with refractive index of 1.49 and the cladding is a fluorinated polymer with refractive index of 1.4. The light attenuation of the fiber is given as being less than $0.2 \mathrm{db} / \mathrm{m}$ for wavelengths between $400-600$ $\mathrm{nm}$. Both ends of the plastic fiber are also polished with the alumina powder. One end of the fiber is attached on the $4.9 \times 4.9 \mathrm{~mm}^{2}$ surface of the scintillator piece with an optical cement and then the whole assembly is clad with a thermally shrinkable black tube to block the ambient lights. The other end of the fiber was coupled to a current-type PMT with a fiber adaptor for FC connection. The PMT (Hamamatsu H6780-4) is a modular type with a high voltage supply integrated into the unit. The spectral response of the PMT is $185-850 \mathrm{~nm}$ and the peak sensitivity is at $400 \mathrm{~nm}$. The input voltage to the module is $+15 \mathrm{~V}$. The gain of the PMT is controlled between $10^{2}$ and $10^{6}$ by adjusting the anode control voltage between 0.25 and 0.9 volt. The PMT module is coupled with the data acquisition unit (Hamamatsu C8908) through 3 cables for current signal output, power supply input and control voltage input. The low voltage power supply unit supplies $+15 \mathrm{~V}$ and $+5 \mathrm{~V}$ to the data acquisition unit for the PMT power and the gain control, respectively. The data acquisition unit is connected to the PC with an RS-232 cable through which digitized current signals are transferred to the PC and several control parameters, such as the gain control voltage and charge integration time, are transferred to the unit. The data acquisition and control of the PMT module are actually performed by an operating program installed in the PC. The program is only run in DOS mode. Each current datum averaged over the charge integration time is stored in a file during the user specified time. A dummy fiber of the same kind and length is also prepared without the scintillator tip to eliminate the contribution of the Cerenkov radiation generated in the fiber core.

\section{Methods of dosimeter evaluation}

For the dosimeter model to work properly, the amount of lights generated in the scintillator, which is to be measured as the PMT current, should be proportional to the absorbed energy in the scintillator. The dosimeter model is tested in a panoramic ${ }^{60} \mathrm{Co}$ irradiation chamber with an activity of about $222 \mathrm{TBq}(6,000 \mathrm{Ci})$. A detailed description of the irradiator is found in reference 5 . The detector assembly is inserted into the irradiation chamber, and the PMT current is measured at a number of positions along a radial line from the source Measured positions are $20 \mathrm{~cm}, 25 \mathrm{~cm}, 30 \mathrm{~cm}, 35 \mathrm{~cm}, 40 \mathrm{~cm}$, $60 \mathrm{~cm}, 80 \mathrm{~cm}, 100 \mathrm{~cm}$ and $120 \mathrm{~cm}$ from the source and a measuring time of 60 seconds is used at each position. The PMT gain control voltage was adjusted to 0.3 volt and the charge integration time was set to $1 \mathrm{~s}$. The operating program for the data acquisition unit records the PMT current at every second in a file. As shown in Fig. 2, the dummy fiber is also inserted into the chamber together with the sensor assembly. The PMT current from the dummy fiber is recorded in a different file. After all the measurements are completed, the PMT current from the dummy fiber is subtracted from the dosimeter current data to obtain the net PMT current.

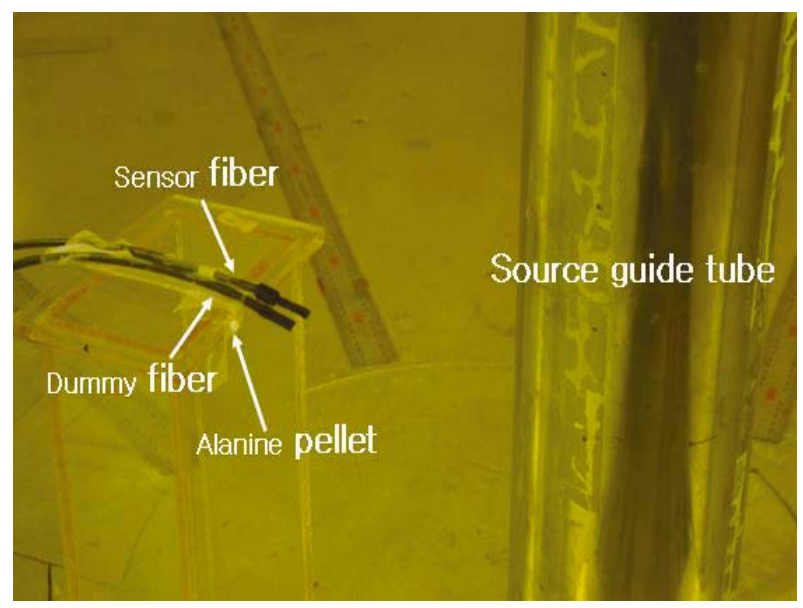

Fig. 2 Arrangements of the sensor fiber, dummy fiber and alanine pellet 
In order to evaluate how accurately the dosimeter model would measure the varying absorbed dose rate as the source-to-dosimeter distance changes, an MCNPX simulation is conducted at each position to calculate the energy deposited in the scintillator volume. In addition, alanine pellet dosimeters supplied by Bruker BioSpin are also employed to measure the absorbed dose rates at the same positions. The alanine dosimeters have been selected since they have higher spatial resolutions and are more accurate in high dose ranges than other dosimeters. Fig. 2 shows the arrangement of the sensor fiber, dummy fiber and alanine pellet fixed on the irradiation stand made of an acrylic glass. The measured absorbed dose rate varied from about 50 to $1,200 \mathrm{~Gy} / \mathrm{h}$ along the measured positions. Since a direct comparison of the PMT current distribution and the deposited energy distribution or the absorbed dose rate distribution can not be done on the same scale, normalized distributions are compared. The normalization is made with respect to the maximum value of each distribution.

A dose rate conversion factor is obtained at each dose rate by dividing the alanine-measured dose rate by the PMT current, and an average conversion factor is obtained over the entire dose rate range. A maximum deviation from the actual dose rates is estimated, that would be expected when the average dose conversion factor is applied. As a means of estimating the effect of a large dose to the scintillator piece, a $100 \mathrm{kGy}$ gamma ray dose is delivered to the scintillator piece. Changes of the light output are measured as the PMT current immediately and one day after the irradiation.

\section{Results and discussion}

Figure 3 shows the time variation of the PMT current measured at $20 \mathrm{~cm}$ from the source for the initial 50 measurements. It can be seen that the fluctuation of the PMT current is very small, and dosimeter model produces very stable signals. This implies that the dosimeter model works reliably. The PMT current from the dummy fiber was about $5 \%$ of the sensor PMT current, and it slightly increased as the sensor was getting closer to the source. Figure 4 compares the normalized distributions of the measured PMT current and energy deposition calculated by MCNPX code. It can be seen that although a considerable deviation exists between the two distributions, the general profiles of the two distributions are similar. Figure 5 compares the normalized distributions of the PMT current and the absorbed dose rate measured with the alanine dosimeters. The two distributions show a minor deviation. This implies that if a proper calibration is done for the dosimeter model, the PMT current can be converted into the absorbed dose rate.

Figure 6 shows the distribution of the dose conversion factors obtained by dividing the absorbed dose rate measured with the alanine dosimeter by the PMT current at each position. Also shown is an average value of the point-wise dose conversion factors. The minimum value is $4.55 \times 10^{5}$ $\mathrm{Gy} / \mathrm{C}$ at $51 \mathrm{~Gy} / \mathrm{h}$ (at position $120 \mathrm{~cm}$ from the source) and the maximum value is $5.54 \times 10^{5} \mathrm{~Gy} / \mathrm{C}$ at $480 \mathrm{~Gy} / \mathrm{h}$ (at

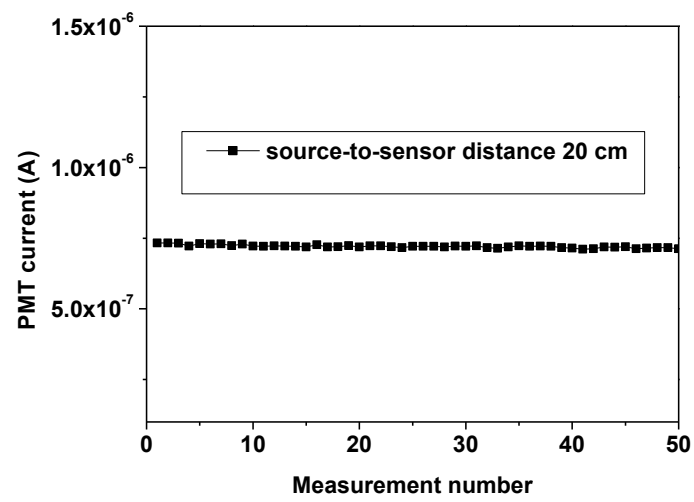

Fig. 3 Time variation of the measured PMT current

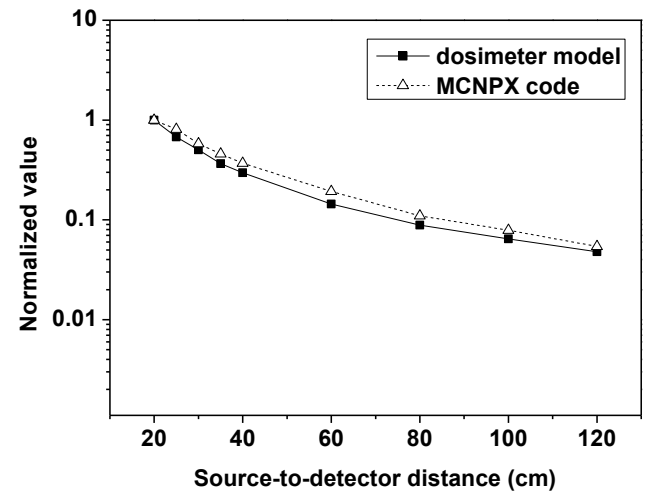

Fig. 4 Comparison of the normalized distribution of the PMT current and MCNPX-calculated deposited energy

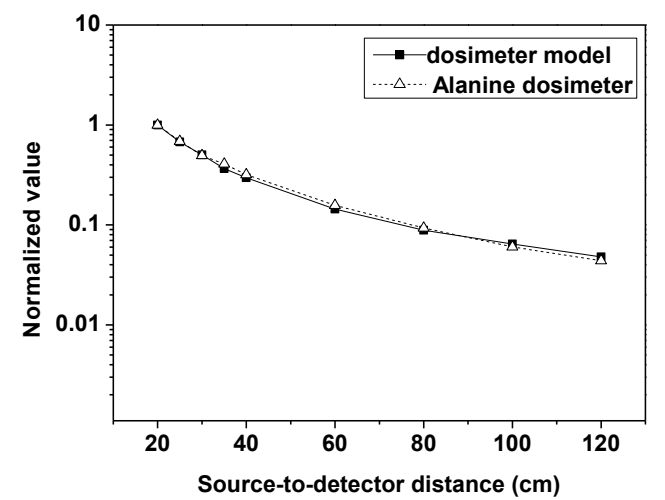

Fig. 5 Comparison of the normalized distributions of the PMT current and dose rate measured with the Alanine dosimeter

position $35 \mathrm{~cm}$ from the source), the average being $5.07 \times 10^{5}$ Gy/C. Figure 7 shows the estimated deviation of the dose rates converted from the measured PMT currents using the average dose conversion factor. A negative deviation indicates a measured dose rate lower than the dose rate measured with the alanine dosimeter, while a positive deviation indicates a higher measured dose rate. The whole deviation stays within $\pm 10 \%$. Considering that there even 


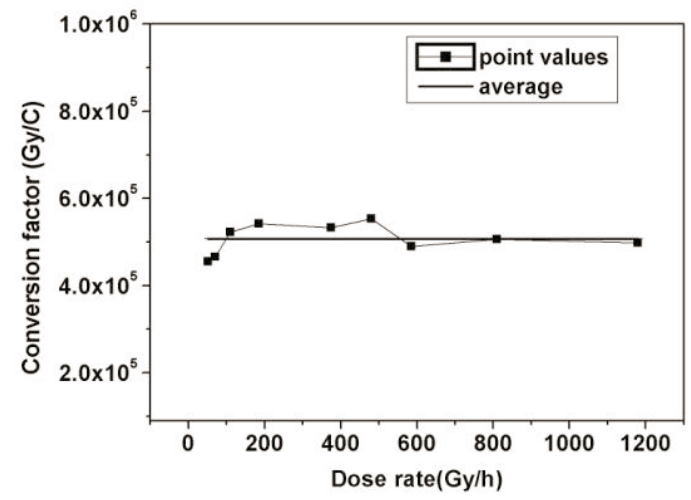

Fig. 6 Dose conversion factors to gain the absorbed dose rate from the measured PMT current. Reference dose rates are measured with alanine dosimeters

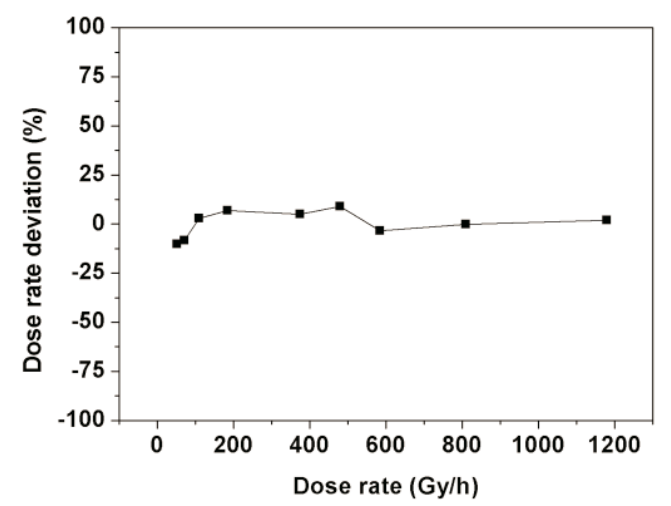

Fig. 7 Estimated deviation of the absorbed dose rates converted from measured the PMT current and the average conversion factor

exists about this much deviation between the dose rates measured with commercial dosimeters in high dose ranges, our result is not disappointing.

Figure 8 shows the change of the light output as measured by the PMT current at $20 \mathrm{~cm}$ from the source immediately and one day after a dose of $100 \mathrm{kGy}$ has been delivered to the scintillator piece with a dose rate of about $4,430 \mathrm{~Gy} / \mathrm{h}$. The irradiation was conducted in the same irradiation chamber by placing the scintillator piece almost in contact with the source guide tube. The light output decreases to about $83 \%$ of the normal value just after the irradiation and recovers to $95 \%$ one day after the irradiation. Although the result is not presented here, the PMT current change was negligible after a dose of $10 \mathrm{kGy}$ is delivered to the scintillator piece.

\section{Conclusion}

A fiber-optic scintillation dosimeter model, composed of a small piece of BGO scintillator piece, a low attenuation plastic optical fiber and a current type PMT, was constructed and tested in a ${ }^{60} \mathrm{Co}$ irradiation chamber to evaluate its usability as a photon dosimeter in the irradiation chamber. In comparison with the MCNPX simulations, the dosimeter did not produce satisfactory results. However, in comparison with commercially available alanine pellet dosimeters it showed a satisfactory performance. It is found that the measured PMT can be converted to the absorbed dose rate within an acceptable deviation. The PMT current decreased to some extent after a very large dose has been delivered to the scintillator piece but recovered in short period. Since normal use of the dosimeter does not cause such a high radiation dose, radiation degradation would not be a concern. By improving the sill-incomplete coupling of the scintillator and the fiber optic, the dosimeter model will produce better results. Also, by replacing the sensor tip and light guiding

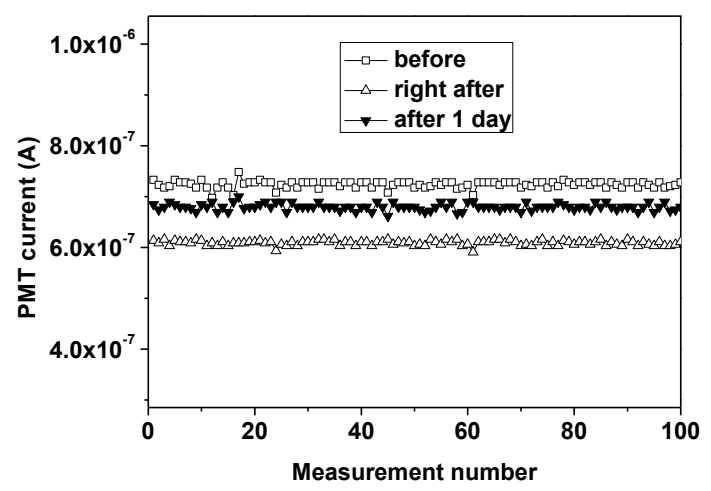

Fig. 8 Change of the light output of scintillator tip before and after ${ }^{60} \mathrm{Co}$ gamma-ray irradiation with dose of $100 \mathrm{kGy}$

optical fiber with materials of higher radiation hardness, a more robust system will be constructed.

\section{Acknowledgment}

This work was performed under the program of Basic Atomic Energy Research Institute (BAERI) which is a part of Nuclear R\&D Programs sponsored by Korea Ministry of Education, Science and Technology

\section{References}

1) R. Nowotny, "LiF:W as a scintillator for dosimetry in diagnostic radiology", Phys. Med. Biol. 49, 2599 (2004).

2) B.S. Lee, Y.M. Hwang, H.S. Cho, S. Kim, and S. Cho, "Fabrication of Fiber-Optic Radiation Sensor Tips with Inorganic Scintillator for Remote Sensing of X or $\gamma$ - ray", IEEE 2, 865(2004)

3) A.S. Bedder, T.J. Kinsella, A. Ikhlef, H. Sibata, "A Miniature Scintillator-Fiberoptic-PMT Detector System for the Dosimetry of Small Fields in Stereotactic Radiosurgery", IEEE Trans. Nucl. Sci., 48 [3], 924 (2001).

4) Y. H. Kim, M. W. Seo, J. W. Park, "A Feasibility Study for the Use of BGO Scintillator in Conjunction with a Plastic Optical Fiber for Remote Gamma Ray Dosimetry", J. Nuci. Sci. Technol. Supplement 5, 542 (2008).

5) Y. H. Kim and J. W. Park, "Dose rate simulation of a panoramic gamma irradiator using the MCNPX code and comparison with measurements", J. Nuci. Sci. Technol. Supplement 5, $325(2008)$. 